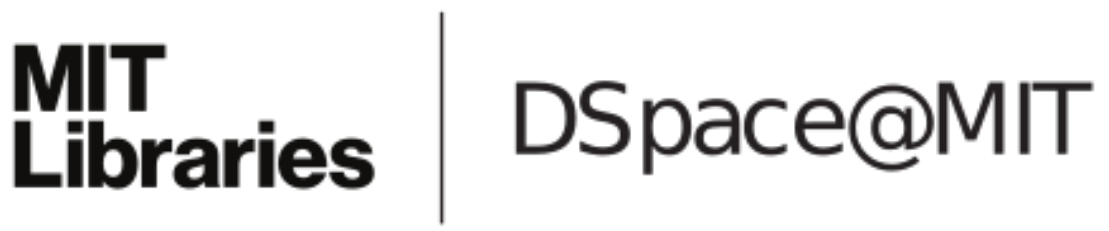

\author{
MIT Open Access Articles
}

Improving rover mobility through traction control: simulating rovers on the Moon

The MIT Faculty has made this article openly available. Please share how this access benefits you. Your story matters.

As Published: https://doi.org/10.1007/s10514-019-09846-3

Publisher: Springer US

Persistent URL: https://hdl.handle.net/1721.1/131905

Version: Author's final manuscript: final author's manuscript post peer review, without publisher's formatting or copy editing

Terms of Use: Article is made available in accordance with the publisher's policy and may be subject to US copyright law. Please refer to the publisher's site for terms of use. 


\section{Improving rover mobility through traction control: simulating rovers on the Moon}

Cite this article as: Ramon Gonzalez, Dimi Apostolopoulos and Karl Iagnemma, Improving rover mobility through traction control: simulating rovers on the Moon, Autonomous Robots https://doi.org/10.1007/s10514-019-09846-3

This Author Accepted Manuscript is a PDF file of an unedited peer-reviewed manuscript that has been accepted for publication but has not been copyedited or corrected. The official version of record that is published in the journal is kept up to date and so may therefore differ from this version.

Terms of use and reuse: academic research for non-commercial purposes, see here for full terms. https://www.springer.com/aam-terms-v1 


\title{
Improving rover mobility through traction control. Simulating rovers on the Moon
}

\author{
Ramon Gonzalez ${ }^{1,2, *}$, Dimi Apostolopoulos ${ }^{3}$, Karl Iagnemma ${ }^{2}$ \\ ${ }^{1}$ Massachusetts Institute of Technology, 77 Massachusetts Ave, 02139, Cambridge, MA \\ ${ }^{2}$ robonity: innovation-driven startup, 5 Extremadura Street, 04740, Roquetas de Mar, Almeria, Spain \\ ${ }^{3}$ ProtoInnovations LLC, 100 43rd Street, 15201, Pittsburgh, PA
}

This paper shows the performance of various traction control strategies that aim to minimize slippage and wheel fighting by properly adjusting the velocity of each traction wheel in a planetary rover. These strategies are validated through simulations performed in $A N$ VEL (Quantum Signal LLC) and using two rovers currently employed by NASA. These experiments use similar features to those that a planetary rover would face on the Moon such as terrain geomorphology and lunar gravity. After running those experiments, the following conclusions were drawn: (1) when no traction control is considered, results show the rover gets entrapped or makes a shorter progress than when traction control is applied; (2) the proposed traction controllers demonstrate a proper balance between slipcompensation (lowest mean slip) and reduction of wheel fighting effects (less aggressive control actions); (3) after considering two different planetary rovers, it is observed that the mechanical configuration effects slip reduction. These contributions can also be observed in the accompanying videos.

Keywords: kinematic incompatibility, K-REX rover, Moon South Pole DEM, slip compensation, theia rover

\footnotetext{
${ }^{*}$ Corresponding author: ramon@robonity.com
} 


\section{INTRODUCTION}

What makes Mars and the Moon interesting to scientists also makes them challenging to a planetary exploration rover: their unique and challenging terrain [Gonzalez and Iagnemma, 2017]. Steep terrain mobility is not only limited to hard surfaces, but also to loose materials at an angle of response (e.g. dunes, slopes). Though advanced Mars rover designs have been developed for traversing challenging terrain conditions, Mars rovers still experience mobility-related issues. For instance, on sol 461 (April 26, 2005), the NASA's Opportunity rover got stuck in a sand dune in Meridiani Planum (http://mars.nasa.gov/mer/ missionstatus_opportunityAll_2005.html). It took five weeks for the engineers to extract it from this situation. The Curiosity rover has also faced challenging situations comprising soft soils (Parnell, 2015).

There is a broad body of literature solving two of the main problems involving off-road robots. The first problem deals with minimizing the soil embedding risk or maximizing the traction, those issues are directly related to slip. In this context, some approaches try to avoid slip generating control signals such that the soil never fails (Iagnemma \& Dubowsky, 2004; Lamon \& Siegwart, 2007). These approaches rely on complicated torque-based traction controllers, which involve numerous parameters that are difficult to measure real-time. Other researchers propose simpler velocity-based slip-compensation controllers (Gonzalez, Rodriguez, \& Guzman, 2014; Helmick et al., 2006). These control strategies adapt the control signals depending on the estimated slip. The main limitation of this paradigm is that in general slip cannot be accurately estimated in a continuous way (Gonzalez, Apostolopoulos, \& Iagnemma, 2018; Iagnemma \& Ward, 2009).

One of the main drawbacks of the previous strategies, either avoiding slip or compensating slip, is that they do not account for the second major problem related to the mobility of mobile robots in off-road conditions, that is, the wheel "fighting" phenomenon or kinematic incompatibility. This phenomenon is a natural outcome of rover travel over uneven terrain, it explains why the wheels may move at different velocities, which ultimately means a lack of coordination among them. There are not many references solving this issue in the field of mobile robotics (Baumgartner, Aghazarian, Trebi-Ollennu, Huntsberger, \& Garrett, 2000; Peynot \& Lacroix, 2003). However, this point constitutes a known problem in the automotive field and several techniques have appeared such as ABS (Anti-lock Braking System) and ESP (Electronic Stability Program) (Ulsoy, Peng, \& Cakmakci, 2012). However, these approaches do not take into account the slip derived from the vehicle-terrain interaction (the kind of slip that appears in planetary exploration rovers).

The solution proposed here not only aims at reducing wheel slip by taking (discrete) slip estimates, but also, reduces wheel "fighting" (kinematic incompatibility) by coordinating the velocity among multiple rover wheels. The performance of the proposed strategies is validated 
using the advanced robotic simulator ANVEL (Quantum Signal) with a new planetary exploration rover designed by the authors and called theia rover. This rover is inspired by the new rover under development by NASA for future missions to the Moon (Andrews, Colaprete, Quinn, Bluethmann, \& Trimble, 2015). Additionally, a model of the NASA's K-REX rover (planetary exploration rover assembled by ProtoInnovations for NASA AMES) has been also used for comparison purposes. In order to get realistic and meaningful conclusions, a real DEM of the South Pole of the Moon has been imported into ANVEL (Moon LRO South Pole DEM). The simulations have been tuned according to the lunar environment (e.g. lunar gravity, lunar terrain properties, etc.).

This paper is organized as follows. Section 2 presents the traction control strategies proposed in this work. Section 3 provides experimental results showing the performance of the traction control algorithms. Section 4 deeply discusses the results obtained in this research. The steps to be followed to validate the simulated results through physical experiments are highlighted in Section 5. Section 6 concludes the paper and summarizes future efforts. Videos with the simulated experiments are available at: https://youtu.be/gyQtb_WcmAY

\section{TRACTION CONTROL STRATEGIES}

Traction control constitutes a standard in the automobile community, which is designed to prevent loss of traction of driven road vehicles (under acceleration). When the traction-control system determines that one wheel is spinning more quickly than the others, it automatically "pumps" the brake to that wheel to reduce its speed and lessen wheel slip (Rosenbluth, 2001). This work uses a different model to approach the traction control problem. Here, the rover does not lose traction (slippage) because of acceleration. Slippage is produced by the nature of the terrain (soil failure). Thus, reducing the velocity may cause the rover to sink and become trapped.

Soil failure: in the context of planetary exploration rovers is defined as the critical situation experienced by a rover when the intensity of loading over a soil exceeds the safe bearing capacity of such soil. This situation leads to rover slippage or even rover entrapment.

\subsection{Velocity-based traction control for slippage compensation}

Generally, the traction control problem can be stated as follows: Given a desired body forward velocity, $v_{b o d y}$, compute the angular velocity of each wheel, $\omega_{j}, j=1 \ldots N_{w}$, such that the no slip condition is satisfied for an arbitrary robot pose and ground contact state. Notice that this approach differs from traditional traction control methods involving torque (Gonzalez \& Iagnemma, 2018; Iagnemma \& Dubowsky, 2004; Krebs, Thueer, Carrasco, \& Siegwart, 2008). The 
main motivation why this paper considers velocity-based traction control is that torque-based strategies require numerous terramechanics-related parameters which are difficult to measure or estimate real-time. The velocity-based strategies work by compensating independently the velocity of each wheel considering the desired body velocity (used to synchronize the wheels), and its slip. Testing and analysis of velocity-based traction control, explored more in subsequent sections shows not only the reduced risk of rover entrapment, but also the decrease of average slip values. After deeply analyzing the phenomena involved in the wheel-terrain interaction this result was not unexpected. As reported in (Kim, 1969; Wong \& Reece, 1967), when a wheel is moving on compact sand, the effect of increasing the wheel torque (wheel angular velocity) leads to a higher drawbar pull (wheel thrust - wheel resistance). This phenomenon is also tackled in (Rohani \& Baladi, 1981). The authors show that the resistance to the penetration of a dense sand is higher than in loose sand and is also higher at higher depths. This motivates why increasing the angular velocities of the wheels on dense sand does not lead to higher sinkage.

Following the previous reasoning, four traction control algorithms have been proposed in this paper. The first solution is based on the kinematic relation between the wheels, and its only goal is to minimize the kinematic incompatibility issue while considering the contract angle of the wheels. The second traction control approach not only takes into account the kinematic relation between the wheels, but also, the slip at each wheel. This slip estimate is assumed to be a continuous variable. The third approach means a trade-off between the two previous solutions and needs of a certain threshold to commute between them. The last method minimizes the kinematic incompatibility issue and compensates for slip. This slip value is discretized according to three or four classes (e.g. low slip, moderate slip, and high slip). The methodology proposed for discretizing wheel slippage has already been published in (Gonzalez et al., 2018). This idea is a promising solution because online estimation of longitudinal slip is not reliable for slow-moving rovers (noisy measurements). The use of a discrete variable then represents a much more practical solution, see (Gonzalez \& Iagnemma, 2018) for a deeper discussion.

\subsection{Strategy 1. Kinematic incompatibility}

The first strategy is based on analysis of the kinematic relations between rover wheels (Baumgartner et al., 2000; Peynot \& Lacroix, 2003). Here, a new control input for a wheel is computed when a wheel's contact angle is different than zero. The angular velocity for a wheel can be computed as

$$
v_{j}=\frac{v_{b o d y}}{\cos \left(\gamma_{j}\right)} \rightarrow \omega_{j}=\frac{v_{b o d y}}{R \cos \left(\gamma_{j}\right)}, \forall j=1, \ldots, N_{w}
$$


where $\omega_{j}$ is the control input to wheel $j, R$ is the wheel radius, and $N_{w}$ is the number of wheels. By enforcing this relation, the longitudinal component of the rover wheel velocities is the same. Then, as long as the global motion of the robot $\left(v_{b o d y}\right)$ respects the references, the low-level PID controllers ensure the given set points, and a sensor provides a measure of the contact angle (or the rocker configuration), $\gamma_{j}$; the effective velocity of all the wheels will be the same, reducing the risk of kinematic incompatibility. Figure 1 illustrates this approach.

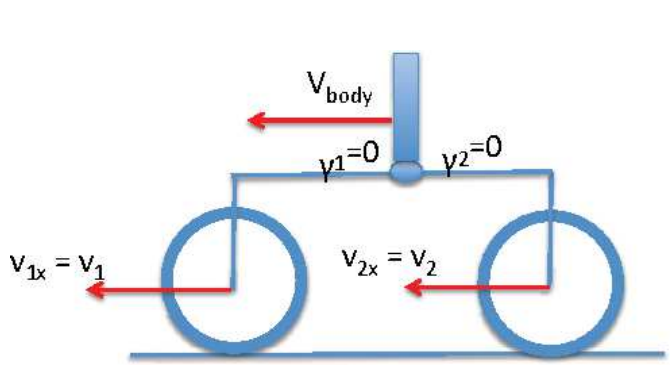

(a) Flat terrain

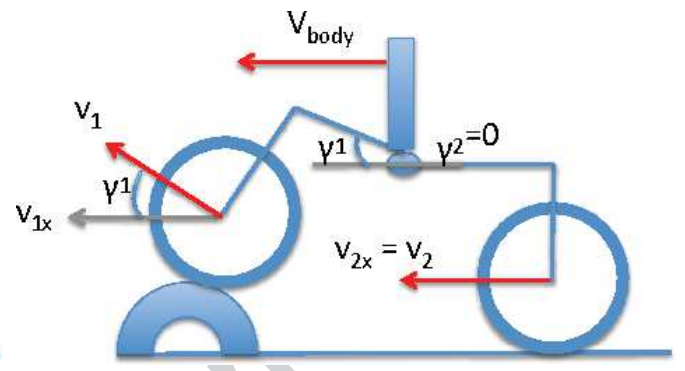

(b) Front wheel moving over a stone

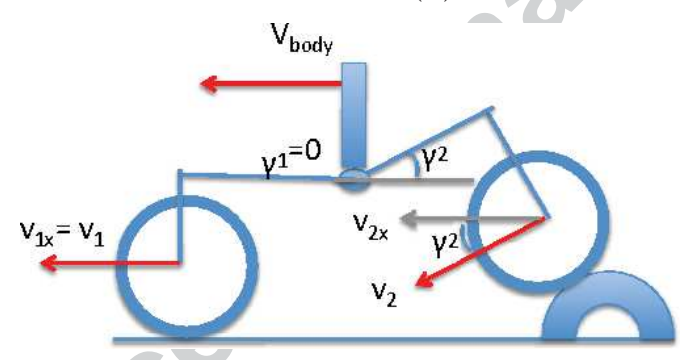

(c) Rear wheel moving over a stone

Figure 1: Strategy 1. Analysis of velocities for traction control compensating the kinematic incompatibility problem (different contact angle). Observe that $v_{j x}$ is the projection in the $\mathrm{x}$-axis of the linear velocity of wheel $j$

\subsection{Strategy 2. Kinematic incompatibility and slip compensation}

The second strategy follows the same general idea of the previous strategy; however, it not only considers the kinematic relation between the wheels, but also considers an estimate of the slip at each wheel (Gonzalez et al., 2018, 2014). In particular, the control input to the wheels is given by

$$
\omega_{j}=\frac{v_{b o d y}}{R\left(1-i_{j}\right) \cos \left(\gamma_{j}\right)}, \forall j=1, \ldots, N_{w}
$$


where $i_{j}$ is the estimated slip of wheel $j$ (continuous value). Furthermore, the new control input is constrained to the actual limits of the motor attached to the wheel, that is, the angular velocity of each wheel cannot exceed a maximum (minimum) value, as in: $\omega^{m} \leq \omega_{j} \leq \omega^{M}$.

\subsection{Strategy 3. Switching policy between kinematic incompatibility and slip compensation}

In Strategy 2, if slip is greater than zero (which is common), there is de-synchronization between the wheels, and corrective control action is needed. This approach may not be necessary or effective if the slip estimates are coarse, or slip values are generally low. This third control strategy therefore only applies the slip-compensation action when slip is greater than a certain threshold, $\delta$. Note that a high value for this threshold can lead to conservative control actions (which can lead to rover embedding). The control inputs are obtained as

$$
\begin{gathered}
\text { IF slip }>\delta \\
\qquad L S E \\
\omega_{j}=\frac{v_{b o d y}}{R\left(1-i_{j}\right) \cos \left(\gamma_{j}\right)}, \forall j=1, \ldots, N_{w}, \\
\omega_{j}=\frac{v_{b o d y}}{R \cos \left(\gamma_{j}\right)}, \forall j=1, \ldots, N_{w} .
\end{gathered}
$$

\subsection{Strategy 4. Switching policy considering discrete slip values}

This new configuration of Strategy 3 considers discrete values for the slip, instead of the continuous feedback of the previous algorithms. Notice that the control action depends on the contact angle (continuous value), the other terms are discrete or constant.

$$
\begin{array}{cc}
\text { IF lowSlip } & \\
\text { ELSEIF moderateSlip } & \omega_{j}=\frac{v_{\text {body }}}{R(1-0.20) \cos \left(\gamma_{j}\right)}, \forall j=1, \ldots, N_{w}, \\
\text { ELSE } & \omega_{j}=\frac{v_{\text {body }}}{R(1-0.46) \cos \left(\gamma_{j}\right)}, \forall j=1, \ldots, N_{w}, \\
& \omega_{j}=\frac{v_{\text {body }}}{R(1-0.83) \cos \left(\gamma_{j}\right)}, \forall j=1, \ldots, N_{w} .
\end{array}
$$

As a first approach based on experiments carried out with a single-wheel testbed at MIT (Gonzalez et al., 2018), three representative values have been used for discretizing the commanded wheel velocity. In this case, the representative value for low slip is $20 \%$ (to be replaced in Eq. 5), the value for moderate slip is $46 \%$ (to be replaced in Eq. 6), and the representative 
value for high slip is $83 \%$ (to be replaced in Eq. 7). Assuming a body velocity of $0.1[\mathrm{~m} / \mathrm{s}]$, the commanded angular velocity for the low-slip case would be around $0.5[\mathrm{rad} / \mathrm{s}]$ (it also depends on the contact angle), for the moderate-slip case around $0.75[\mathrm{rad} / \mathrm{s}]$, and around $2.35[\mathrm{rad} / \mathrm{s}]$ for the high-slip case.

\section{$3 \quad$ RESULTS}

The advanced robotic simulator ANVEL (Quantum Signal LLC) has been used for comparing the performance of the proposed traction control algorithms. ANVEL uses an extended ODE (Open Dynamics Engine) friction model in order to simulate the interaction between the vehicle and the terrain (VTI model). The model-specific properties for the default VTI model include values that ODE uses during its internal collision routines, such as friction in the lateral and longitudinal directions, surface density, cohesion, and internal friction angle, among other variables. In addition to that, the user can adjust the gravity to be simulated. In this case, a lunar regolith terrain has been selected with a surface density of $1500(\mathrm{~kg} / \mathrm{m} 3)$, cohesion of 1 $(\mathrm{kPa})$, internal friction angle of 30deg, and gravity of $1.622 \mathrm{~m} / \mathrm{s}^{2}$ (Moon).

The authors have designed and implemented a model of a planetary exploration rover with independent suspension. The nickname of the new rover is: "theia rover". This rover is based on the RP rover currently under testing by NASA (Andrews et al., 2015). For comparison purposes, a model of K-REX rover has also been used. More information about the K-REX rover can be found in (Gonzalez et al., 2018). Videos with these experiments are available at: https://youtu.be/gyQtb_WcmAY

A real 4-m-resolution DEM of the Moon South Pole has been imported into ANVEL (Moon LRO South Pole DEM). After that, a representative scenario has been searched over the entire DEM. That scenario has been selected according to the traditional challenges faced by planetary rovers like slopes in sandy terrains (Arvidson et al., 2017). In this case, the rover climbs over a slope of sandy/rocky terrain (similar cohesion and internal friction angle than lunar regolith, $c$ $=1 \mathrm{kPa}$ and $\phi=30^{\circ}$ ) (Heiken, Vaniman, \& French, 1991). For these specific simulations, the desired rover velocity was set to $0.1[\mathrm{~m} / \mathrm{s}]$, and the angular velocity of the wheels was constrained to $\{-5.5,5.5\}[\mathrm{rad} / \mathrm{s}]$. Notice that the desired rover velocity is similar to the velocities reached by current Mars rovers (MER and MSL rovers) (Grotzinger et al., 2012). Simulations have been run for 60 seconds and a total of seven experiments have been run with the theia rover and two with the K-REX rover. The selected scenario is displayed in Figure 2.

Two different configurations have been tested of Strategy $3(\delta=0.75$ and $\delta=0.35)$. Strategy 4 has also been tuned according to two different setups. In the first case, slip is discretized according to three classes: class 1 ( slip $\leq 30 \%)$, class $2(30<\operatorname{slip} \leq 60 \%)$, and class 3 (slip $>60 \%$ ). In the second case, slip is discretized according to four classes: class 1 (slip $\leq 20 \%$ ), 


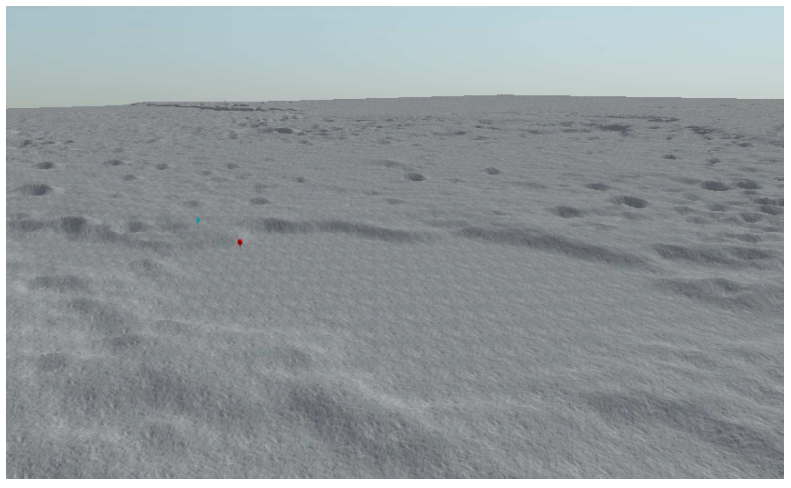

(a) Overhead view (Moon crater)

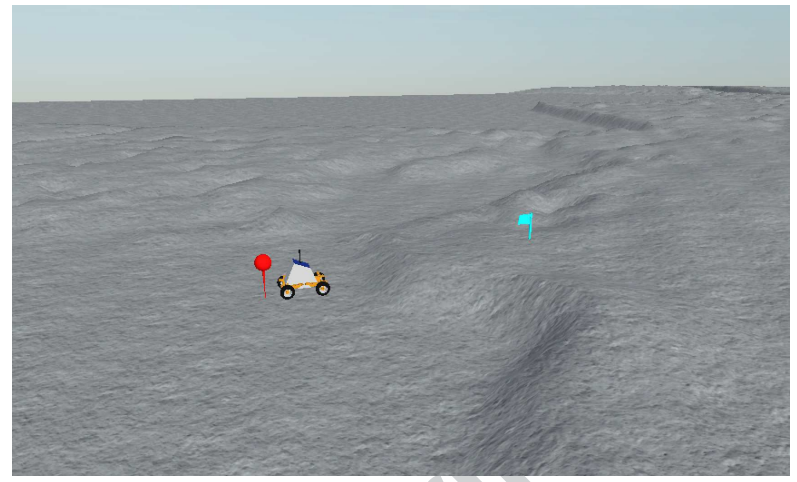

(b) Detail of the environment

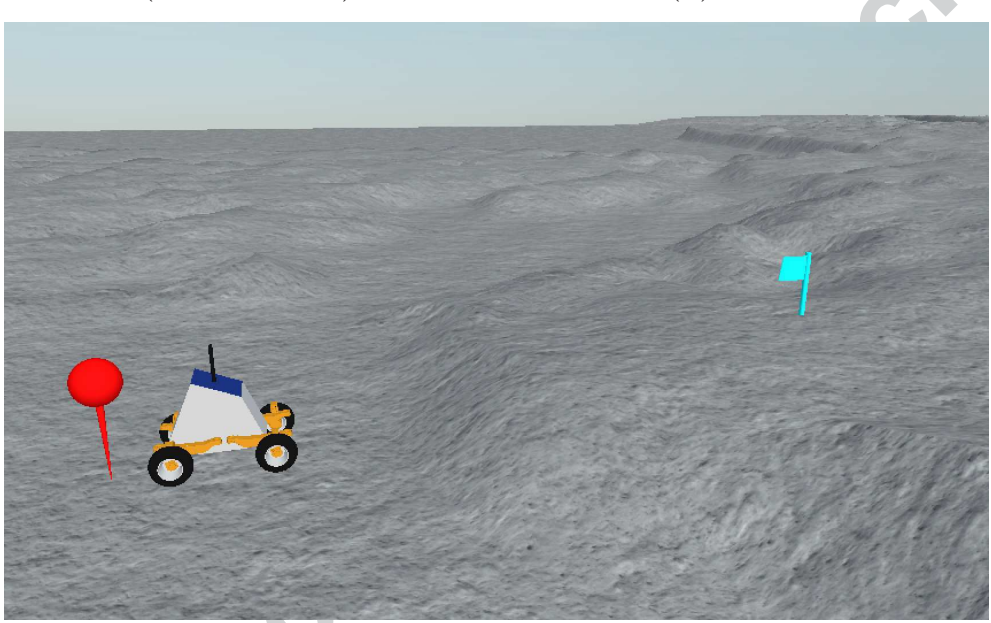

(c) Close view of the environment and theia rover

Figure 2: Scenario considered in this work and planetary exploration rover used for the simulations (theia rover). Notice that the rover travels over dry sandy terrain, which may lead to slip, embedding, and even rover entrapment

class $2(20<\operatorname{slip} \leq 40 \%)$, class $3(40<\operatorname{slip} \leq 60 \%)$, and class 4 (slip $>60 \%)$. In addition to the traction controllers proposed in this paper, the case of no traction control has also been considered for comparison (it is called Strategy 0).

Before addressing the performance of the traction controllers, it is important to point out that no high-level path follower is running while the rover is moving. The traction controllers implemented in these simulations are only responsible for the wheel velocities (not the position of the whole rover). This explains why when the rover deviates from the trajectory, due to the 
uneven nature of the terrain and the slip events, it does not correct such deviation.

Figures 3a, 3c display the trajectories of the rover when various control strategies are employed (alg0: no traction control, alg1: Strategy 1, alg2: Strategy 2, alg3075: Strategy 3/variant $1(\delta=0.75)$, alg3035: Strategy 3/variant $2(\delta=0.35)$, alg3dis $(1)$ : Strategy 4/variant 1 (3 discrete slip classes), and alg3dis (2): Strategy 4/variant 2 (4 discrete slip classes). In most cases theia rover climbs the slope. However, in some cases the rover fails to scale the slope. When no traction control is considered, the rover gets entrapped before reaching the top. Strategy 2 and Strategy 3/variant 2 produce excessively aggressive control actions that force the rover to deviate from the desired straight path. The best result is obtained with the strategies considering discrete slip classes (Strategy 4/variant 1 and Strategy 4/variant 2). Note that in the best cases, the rover not only climbs the slope, but also moves further than Strategy 1 and Strategy 3/variant 2. Recall that the simulation runs for the same amount of time for all the all the experiments and the configuration of the rover is the same throughout all the simulations (except for the traction control algorithms). Figure $3 b$ shows the heading of the theia rover for each traction control strategy. There is a large deviation experienced in Strategy 2 and Strategy 3/variant 2. When the traction controllers based on discrete slip estimates are employed the rover moves further than Strategy 1 and Strategy 3/variant 1 (around 7.5 meters versus 5.5 meters).

Figure 4 shows the control inputs. Observe how the commanded velocity changes depending on the contact angle for every traction control strategy except for the case when no traction control is employed. Strategy 1 and Strategy $3 /$ variant 1 do have a small change in the commanded velocity between the 2nd and 3rd meters. Looking at this figure, it is easy to understand the wrong result obtained while considering Strategy 2 and Strategy 3/variant 2. Recall that these approaches generate the control action according to the current (continuous) value of the slip and the contact angle. It means that even small slip creates de-synchronization among the wheels in the rover. This effect is even augmented along time and distance until a certain point where the rover is uncontrollable. This is exactly what happens after 3 meters from the starting point. In addition to these aspects, it is also interesting to highlight that the commanded control inputs saturate at the maximum velocity achievable by the wheels $(\{-5.5,5.5\}[\mathrm{rad} / \mathrm{s}])$.

Figure 5 shows in detail the behavior of Strategy 4/variant 1. As expected, the control action changes according to three discrete values (the small increments/decrements around those three discrete values are due to the variation in the contact angle). The actual velocities reached by the wheels also match the control inputs generated by the traction controller. This means that the low-level PID controllers, in charge of reaching the desired control inputs, have been tuned properly.

Figure 6 displays the contract angles. Observe the uneven nature of this scenario, because the wheels are always facing a slope of more than 20 degrees.

Finally, Figure 7 shows the estimated slip in terms of distance traveled. When no traction 


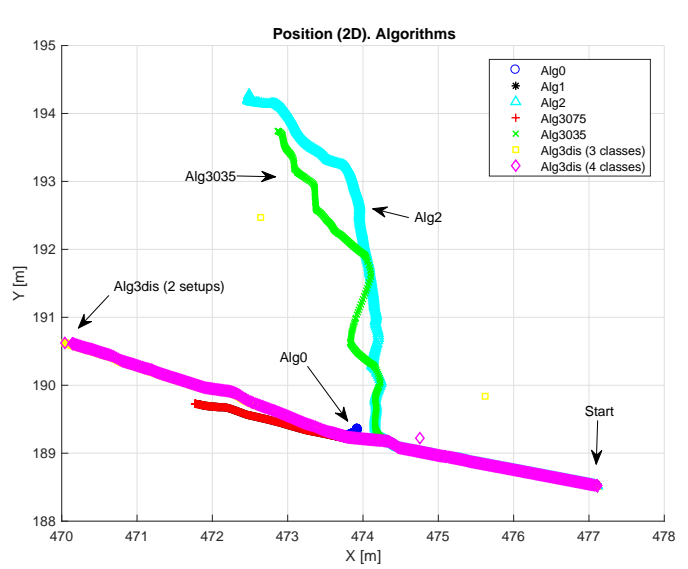

(a) $2 \mathrm{D}$ view of the trajectories

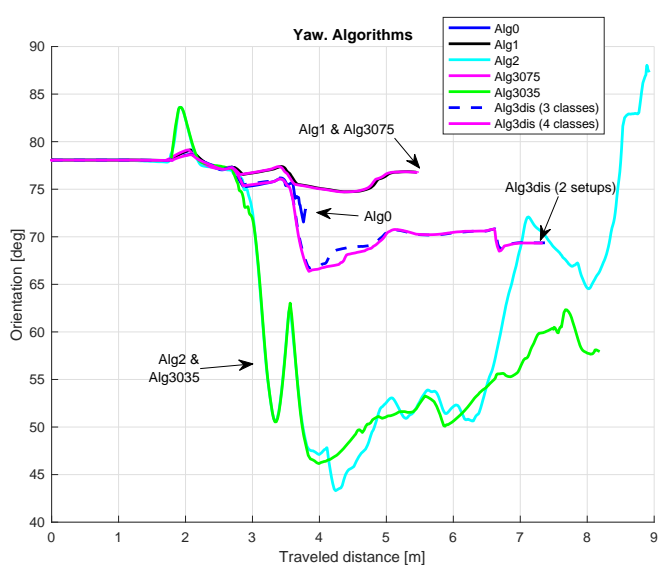

(b) Theia's heading

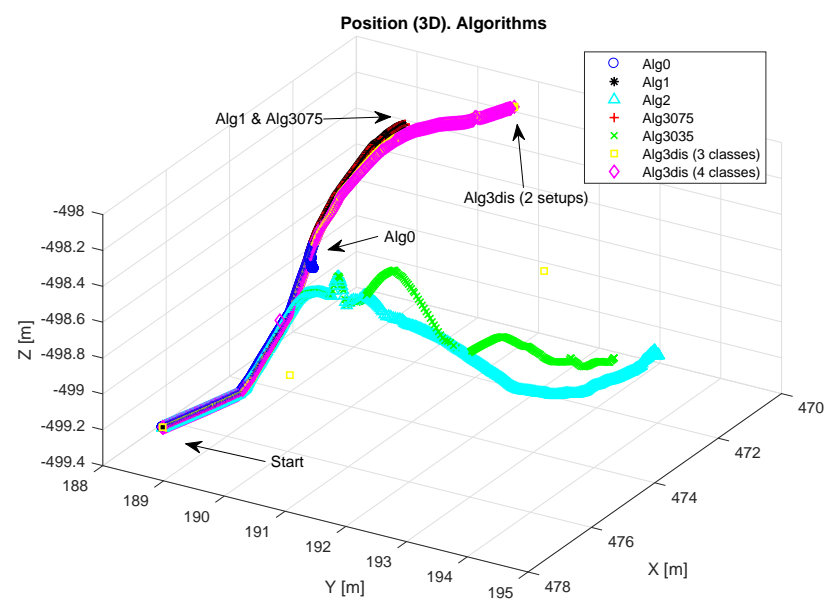

(c) $3 \mathrm{D}$ view of the trajectories

Figure 3: Performance of the traction control algorithms in terms of the tracked trajectory for theia rover. Notice the large deviation of Strategy 2 and Strategy 3/variant 2

control is employed the rover gets stuck ( $\operatorname{slip}=100 \%$ ). Strategy 2 and Strategy 3/variant 2 generate aggressive control actions that not only lead the rover to deviate from the reference path, but also to experience many high-slip events. The lowest average slip is obtained by means of Strategy 1 and Strategy 3/variant 1. The two implementations dealing with discrete slip represent a proper balance between low slip and moderate slip events and the further traveled 

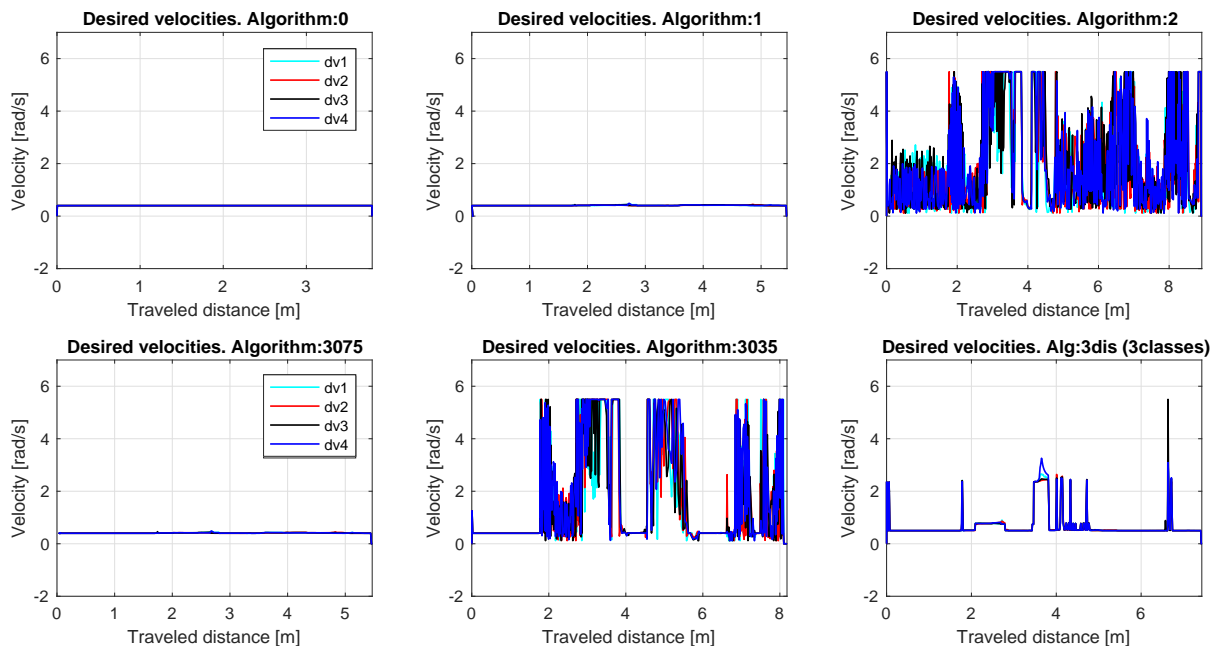

Figure 4: Control inputs (desired velocities) for theia rover. As expected, the control actions generated by Strategy 4 vary within 3 discrete values

distance. 

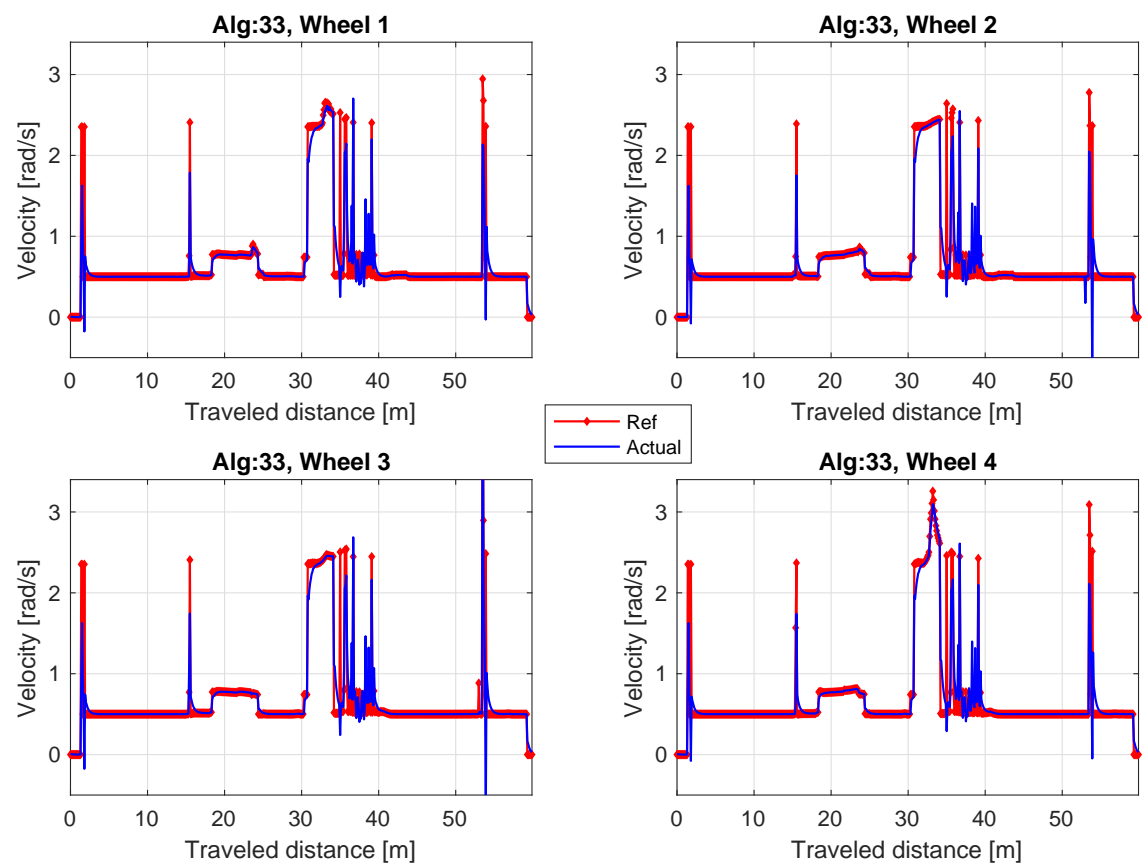

Figure 5: Control inputs versus actual velocities reached by the wheels while running Strategy 4/variant 1 for theia rover. Observe the proper performance of the low-level PID controllers

\section{DISCUSSION}

This section presents a comprehensive comparison of the performance of each traction control strategy while considering the simulations with the theia rover.

Figure 8 shows some statistics related to the traveled distances and the mean absolute errors. When no traction control is considered, it leads to the shortest traveled distance as the rover gets entrapped. Strategy 1 and Strategy 3/variant 2 allow the rover to climb the slope, but mean a shorter trajectory than when Strategy 4 is applied. Recall that, simulations were run for exactly the same time (60 seconds). So, these differences are due to the generated control actions and the slip events. The longest routes are obtained with Strategy 2 and Strategy $3 /$ variant 2. However, those routes deviate largely from the desired path as shown in Figure $8 \mathrm{~b}$ (largest mean absolute error). In Figure 8b, the route followed by Strategy 4/variant 1 has been considered as the reference. Notice that the trajectories followed when the slip is considered as a discrete variable are quite similar and represent the longest and most accurate routes. 

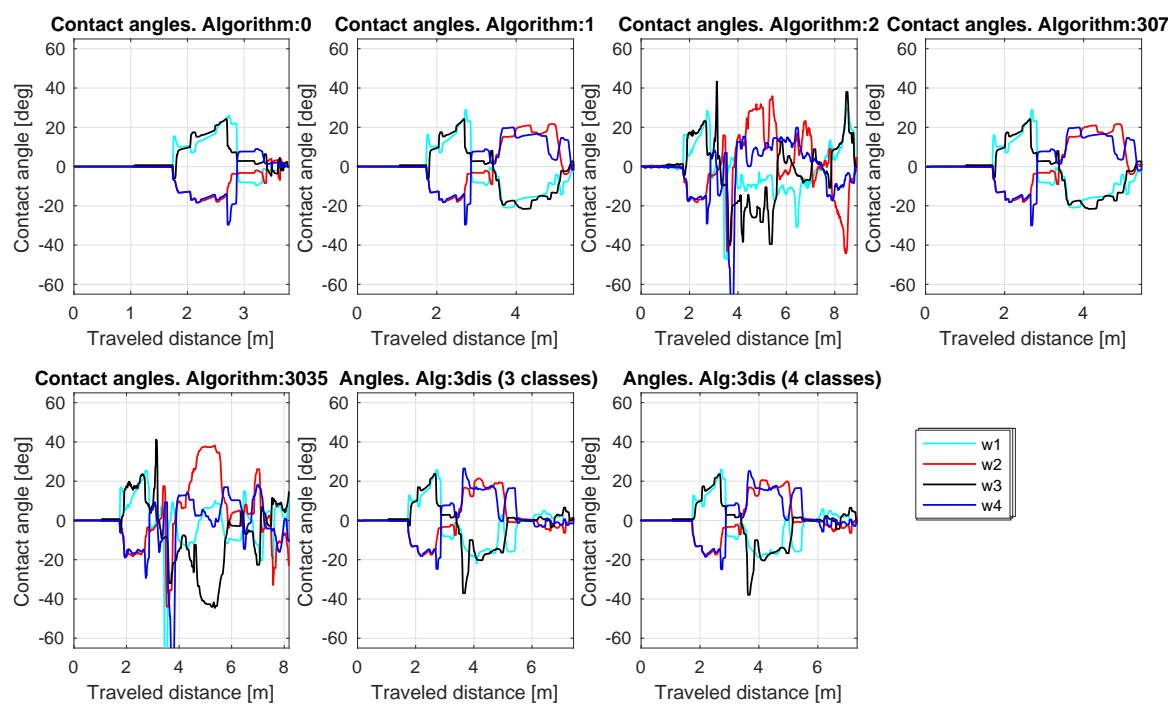

Figure 6: Contact angles for theia rover. These values are expected according to the uneven nature of the environment considered for the simulations
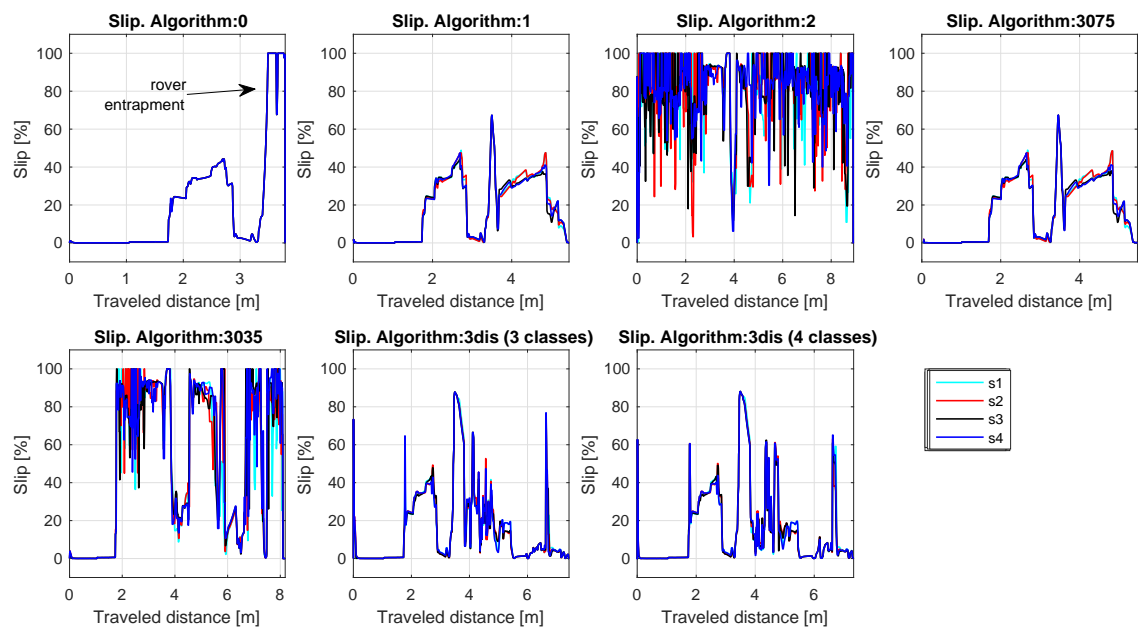

Figure 7: Estimated slip for theia rover. Observe that when no traction control is employed the rover gets entrapped 


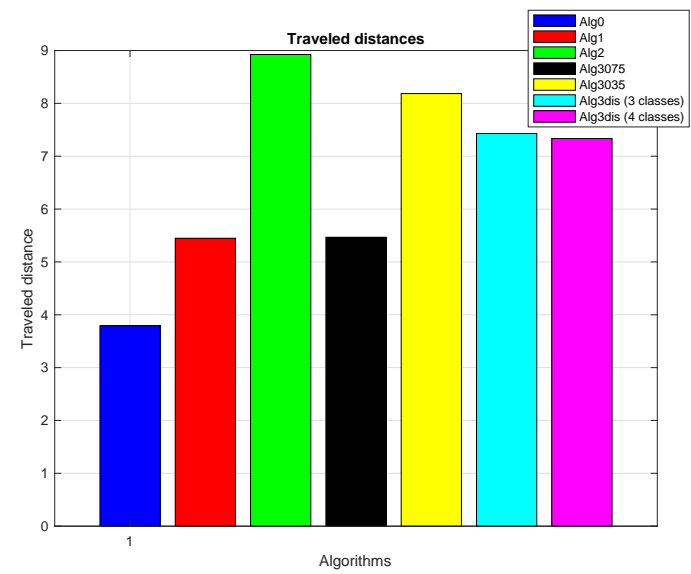

(a) Traveled distances

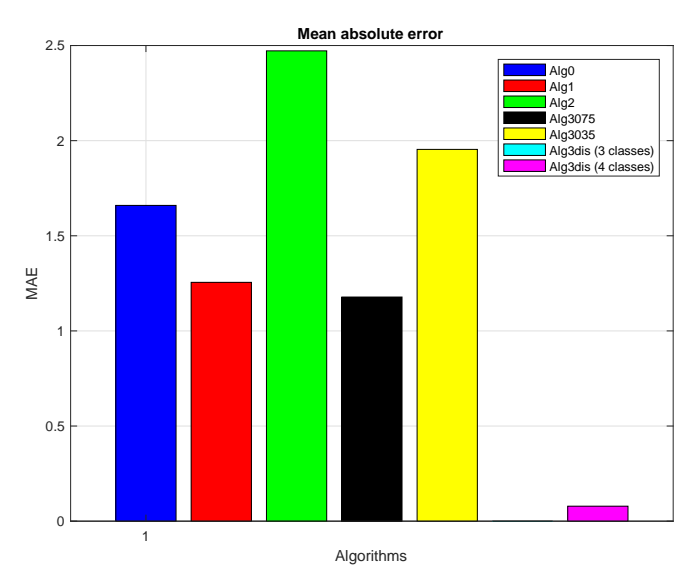

(b) Mean absolute errors

Figure 8: Traveled distances and mean absolute errors reached by the traction control algorithms

Figure 9 shows the histograms with the desired wheel velocities by using Strategy 3/variant 2 and Strategy 4/variant 1. As expected, the wheel velocities comprising the second approach compared here are binned into three groups. In contrast, the velocities generated by Strategy $3 /$ variant 2 span through a wider range. In fact, the second most frequent group is $5.5[\mathrm{rad} / \mathrm{s}]$. This explains the aggressive behavior observed in the slip plot and the deviation from the reference.

The last analysis involves a comparison between theia rover and K-REX rover when both rovers are presented with the same slope climbing task. The two rovers have the same drive and steering configuration and action schemes, but they differ in the suspension type as theia features an independent pivot-arm suspension for each wheel whereas K-REX features a rocker arm-type suspension. As observed in Figure 10, K-REX does not become entrapped in any configuration, even when there is no traction control. Theia rover becomes entrapped when there is no traction control. This is likely because the specific terrain is not as challenging for $\mathrm{K}-\mathrm{REX}$ as is for the theia rover. This observation is reinforced by the fact that the worst-case slip estimates for K-REX when there is no traction control are lower than the slip estimates for theia when a favorable control strategy is employed, see Figure 10c. 


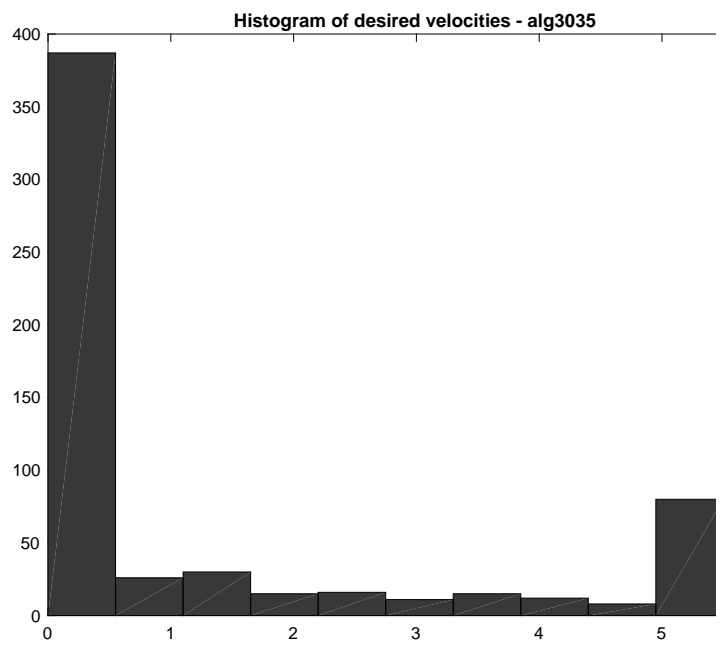

(a) Strategy $3 /$ variant 2

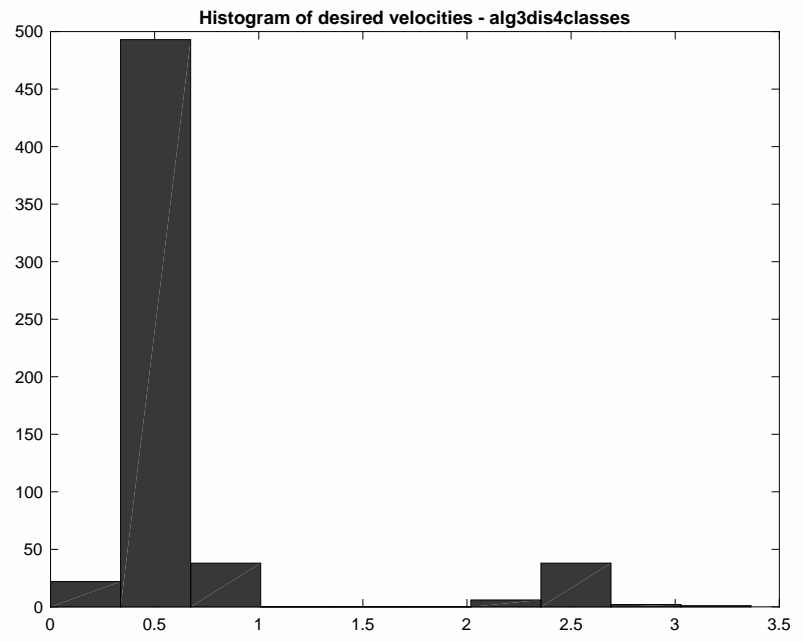

(b) Strategy $4 /$ variant 1

Figure 9: Histograms with the control input generated by the traction controllers

\section{THE PATH TO IMPLEMENTATION}

The proposed control framework will be validated soon on a real planetary exploration rover operating in uneven terrain conditions. Toward that objective, the Lunar All-Terrain Utility Vehicle (LATUV) rover developed by ProtoInnovations LLC in 2009 will be used. Recall that the rover used for simulations, K-REX rover, was also developed by ProtoInnovations and is in service nowadays by NASA. The LATUV rover was developed as part of that effort and comprises a preliminary version of the K-REX rover. This explains why these two rovers share the same mechanical configuration and geometry. As shown in (Gonzalez et al., 2018), the LATUV rover has already been used by the authors in order to tests the performance of slip-detection algorithms through field tests, see Figure 11.

Right now, the authors of this paper and the ProtoInnovations' team are working on updating the entire software architecture of the rover. This effort is taking advantage of the well-known Robot Operating System (ROS) middleware. This new software builds on top of the low-level motor controllers and the sensors. This decision will lead to a more flexible, modular and scalable software architecture. In fact, the authors of this paper are now working on implementing the traction control strategies proposed in this paper as a new module within that architecture. This control module will be fed back by the module responsible for estimating the slippage. Finally, the new control module will be interfaced with the low-level motor controllers. 


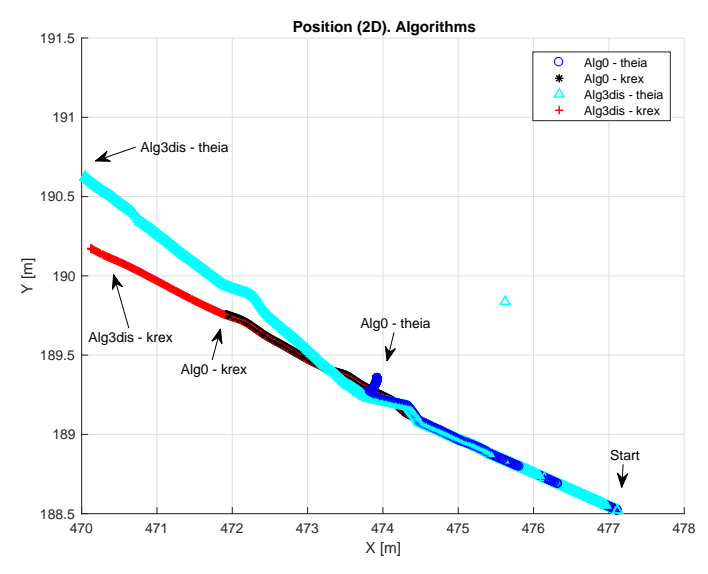

(a) 2D Trajectories

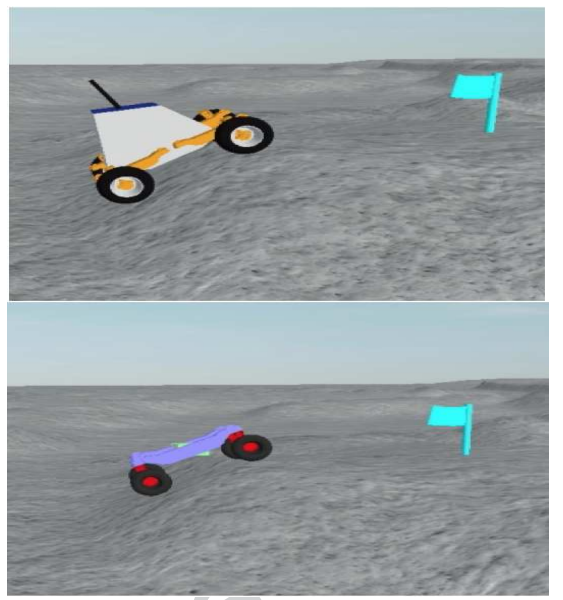

(b) Rovers during the simulation
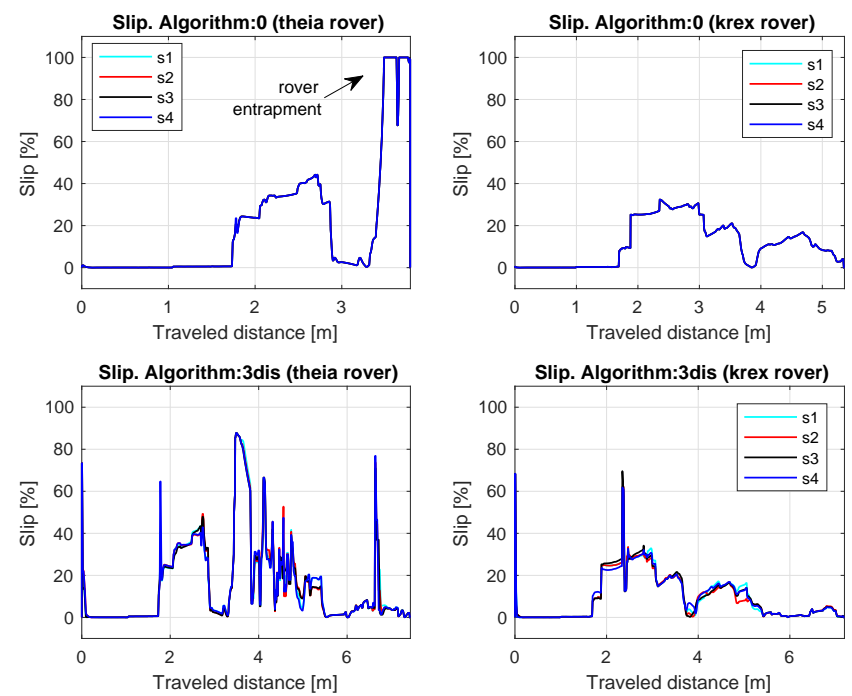

(c) Estimated slip

Figure 10: Trajectories and slip obtained by the theia rover and K-REX rover while using the traction controllers proposed in this paper

Some videos showing the LATUV rover moving in real conditions with no traction control are found at: https://youtu.be/HxjdZfG5b9g. Future experiments using the traction control strategies proposed here will be tested on similar environments and terrains. 


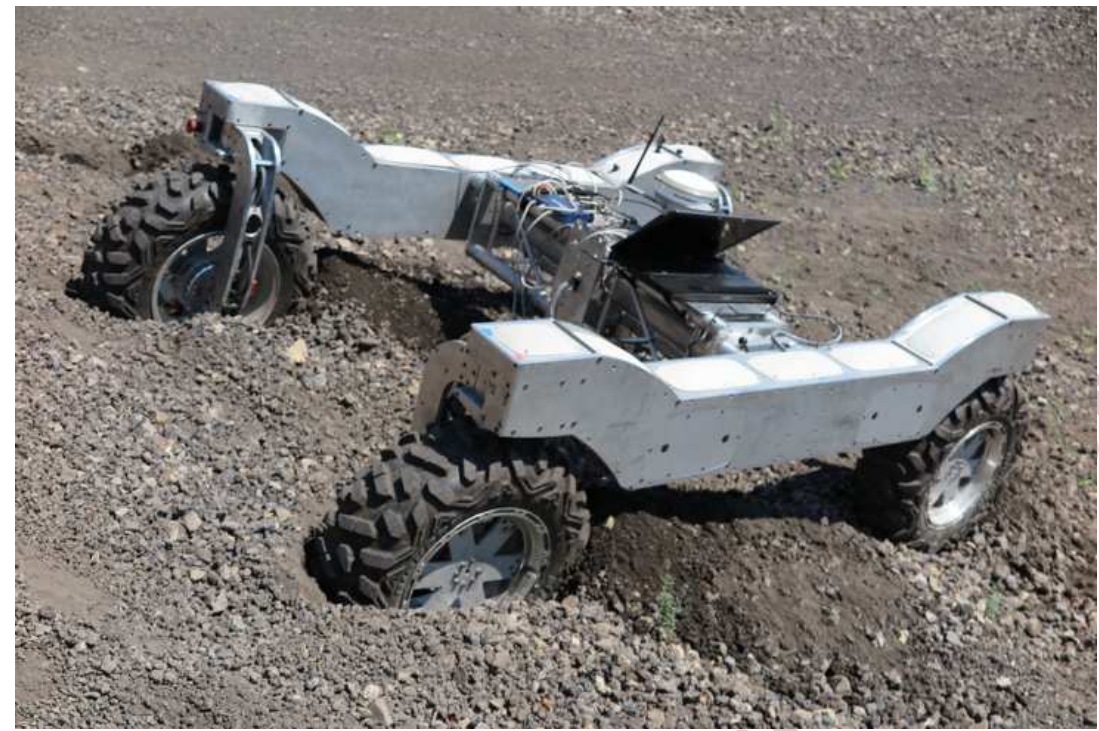

Figure 11: The proposed traction controllers will be validated by using the LATUV rover. This rover has the same mechanical configuration and geometry than the KREX rover used in the simulations

\section{CONCLUSIONS}

The first important conclusion is that the traction controller implemented by a planetary rover impacts the ability for a rover to avoid entrapment and also enhances rover mobility. This is demonstrated by the use of strategies compensating slip and a strategy with no slip compensation at all. In this last case, the theia rover gets trapped in a Moon-like simulation scenario.

This paper comes to confirm that the idea of considering slip as a discrete variable not only demonstrates a proper performance in terms of slip estimation, as already published by the authors in (Gonzalez et al., 2018), but also, in terms of slip compensation.

Another conclusion drawn from this paper is that the mechanical configuration of a planetary exploration rover impacts experienced slip. In simulation the K-REX rover demonstrates better traction capabilities than the theia rover, even when no traction control is applied. This clearly illustrates the effects of rover configuration on terrainability and improvements that can be made by employing the right type of control strategy especially when the rover scales less favorably to the terrain that is negotiating.

Future efforts will focus on validating these traction control strategies through other challenging types of terrains and even new terrain profiles. In addition to that, future research will 
deal with integrating the proposed traction controllers into a complete navigation architecture.

\section{Acknowledgement}

The research described in this publication was carried out at the Massachusetts Institute of Technology (Cambridge, MA) and ProtoInnovations (Pittsburgh, PA), under the STTR Contract NNX15CA25C funded by NASA.

\section{References}

Andrews, D., Colaprete, A., Quinn, J., Bluethmann, B., \& Trimble, J. (2015). Resource Prospector (RP) - Early Prototyping and Development. In Aiaa space conference and exposition. Pasadena, California, USA: AIAA.

Arvidson, R., DeGrosse, P., Grotzinger, J., Heverly, M., Shechet, J., Moreland, S., ... Stilly, E. (2017, October). Relating geologic units and mobility system kinematics contributing to curiosity wheel damage at gale crater, mars. Journal of Terramechanics, 73, $73-93$.

Baumgartner, E., Aghazarian, H., Trebi-Ollennu, A., Huntsberger, T., \& Garrett, M. (2000, October). State Estimation and Vehicle Localization for the FIDO Rover. In G. McKee \& P. Schenker (Eds.), Proc. spie, sensor fusion and decentralized control in robotic systems iii (Vol. 4196).

Gonzalez, R., Apostolopoulos, D., \& Iagnemma, K. (2018, March). Slippage and immobilization detection for planetary exploration rovers via machine learning and proprioceptive sensing. Journal of Field Robotics, 35 (2), 231-247.

Gonzalez, R., \& Iagnemma, K. (2018, June). Slippage estimation and compensation for planetary exploration rovers. State of the art and future challenges. Journal of Field Robotics, $35(4), 564-577$.

Gonzalez, R., Rodriguez, F., \& Guzman, J. L. (2014). Autonomous Tracked Robots in Planar Off-Road Conditions. Modelling, Localization and Motion Control. Springer, Germany.

Grotzinger, J. P., Crisp, J., Vasavada, A., R.C. Anderson, C. B., Barry, R., \& Blake, D. (2012). Mars Science Laboratory Mission and Science Investigation. Space Science Reviews, $170(1), 5-56$.

Heiken, G., Vaniman, D., \& French, B. (Eds.). (1991). Lunar sourcebook. Cambridge University Press.

Helmick, D., Roumeliotis, S., Cheng, Y., Clouse, D., Bajracharya, M., \& Matthies, L. (2006). Slip-compensated Path Following for Planetary Exploration Rovers. Advanced Robotics, $20(11), 1257-1280$. 
Iagnemma, K., \& Dubowsky, S. (2004). Mobile Robots in Rough Terrain. Estimation, Motion Planning, and Control with Application to Planetary Rovers. Springer, Germany.

Iagnemma, K., \& Ward, C. C. (2009). Classification-based Wheel Slip Detection and Detector Fusion for Mobile Robots on Outdoor Terrain. Autonomous Robots, 26(1), 33-46.

Kim, S. (1969). The evaluation of a cone penetrometer as an index of farm vehicle mobility (Unpublished doctoral dissertation). McGill University, Montreal, Quebec, Canada.

Krebs, A., Thueer, T., Carrasco, E., \& Siegwart, R. (2008, February). Towards Torque Control of the CRAB Rover.. (Los Angeles, USA)

Lamon, P., \& Siegwart, R. (2007). 3D Position Tracking in Challenging Terrain. The International Journal of Robotics Research, 26(2), 167-186.

Parnell, B. (2015, May). Slippery slope sends Curiosity rover on a new route across Mars. Forbes Tech.

Peynot, T., \& Lacroix, S. (2003, October). Enhanced Locomotion Control for a Planetary Rover. In IEEE Int. Conf. on Intelligent Robots and Systems (IROS) (Vol. 1, pp. 311 316). IEEE.

Rohani, B., \& Baladi, G. (1981). Correlation of mobility cone index with fundamental engineering properties of soil (Techical Report). US Army Waterways Experiment Station.

Rosenbluth, W. (2001). Investigation and interpretation of black box data in automobiles: a guide to the concepts and formats of computer data in vehicle safety and control systems (Tech. Rep.). ASTM/SAE.

Ulsoy, A., Peng, H., \& Cakmakci, M. (2012). Automotive Control Systems. Cambridge University Press.

Wong, J., \& Reece, A. (1967). Prediction of Rigid Wheel Performance based on the Analysis of Soil-Wheel Stresses. Part I. Performance of Driven Rigid Wheels. Journal of Terramechanics, 4(1), 81-98. 


\section{RAMON GONZALEZ}

Ramon is the founder and CEO of robonity a worldwide tech consulting. He has performed postdoctoral research in the Robotic Mobility Group at the Massachusetts Institute of Technology (3 years), and has been a visiting researcher at the Autonomous Systems Lab (ETH Zurich, Switzerland) and the University of Seville (Spain). He holds a Computer Science Engineering degree and a Ph.D. in mobile robotics from the University of Almeria (Spain). He has also worked at the University of Zaragoza (Spain) as a PhD Assistant. Previously, he worked as a researcher and a lecturer at the University of Almeria. He has also performed a research stay at the ASL-ETH (Zurich, Switzerland). He is author of the monograph Autonomous Tracked Mobile Robots in Planar Off-Road Conditions (Springer, 2014). His main research interests include: modelling, localization and motion control of mobile robots in off-road conditions; terrain classification; computer vision and machine learning.; adaptive, predictive, and robust control; and geostatistics applied to mobile robotics. Ramon is a member of the IEEE Robotics and Automation Society.

\section{DIMI APOSTOLOPOULOS}

Dr. Dimitrios (Dimi) Apostolopoulos' research focuses on using robotics for discovery and exploration, and as a workforce for hazardous duty and in demanding applications. His expertise covers robotic mobility, mechanisms, robot configuration and design, mechatronics, mechanics, dynamics and control. Dr. Apostolopoulos joined NREC in 1998.

Dr. Apostolopoulos holds a Ph.D. in Robotics from Carnegie Mellon University.

\section{KARL IAGNEMMA}

Karl lagnemma is the President of nuTonomy. Before that, Karl was the principal research scientist in the Mechanical Engineering department at the Massachusetts Institute of Technology. He holds a B.S. from the University of Michigan, and an M.S. and Ph.D. from MIT, where he was a National Science Foundation Graduate Fellow. He has performed postdoctoral research at MIT, and has been a visiting researcher at the NASA Jet Propulsion Laboratory and the National Technical University of Athens (Greece). He currently serves on the editorial board of the IEEE Transactions on Robotics and the Journal of Field Robotics. Dr. lagnemma's primary research interests are in the areas of design, sensing, motion planning, and control of mobile robots in outdoor terrain, including modeling and analysis of robot-terrain interaction. He is author of the monograph Mobile Robots in Rough Terrain: Estimation, Planning and Control with Application to Planetary Rovers (Springer, 2004). He has recently led research programs for agencies including the U.S. Army Tank-Automotive and Armaments Command, the Army Research Office, DARPA, the NASA Mars Program Office, Ford Motor Company, and the NASA Institute for Advanced Concepts, among others. He has authored or co-authored many conference and journal papers on a wide range of robotic topics, and has consulted for various private companies and government agencies. Dr. lagnemma was chosen as one of 16 people who redefined science in 2003 by SEED magazine. 
He was a 2005 nominee for a MacArthur Foundation fellowship. 


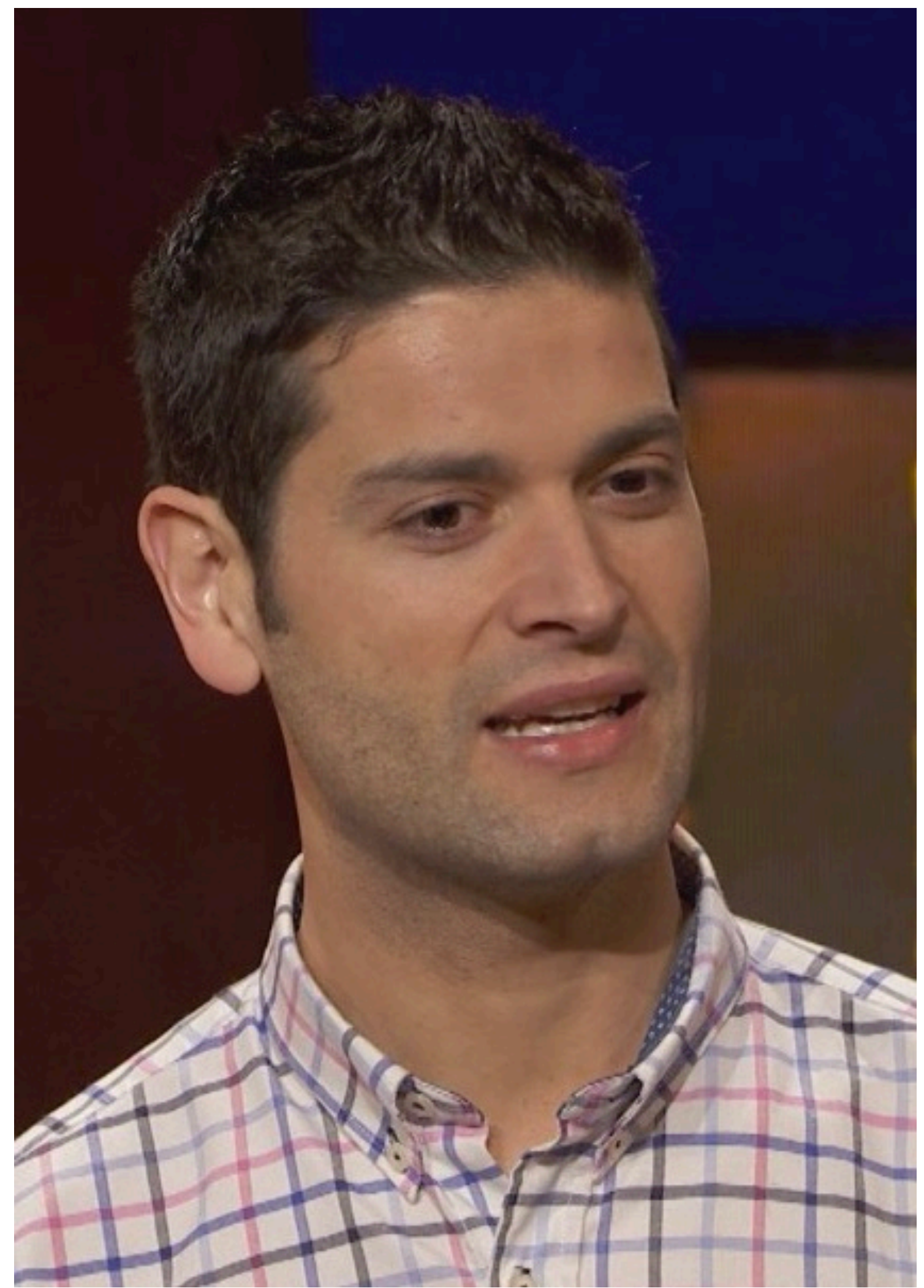




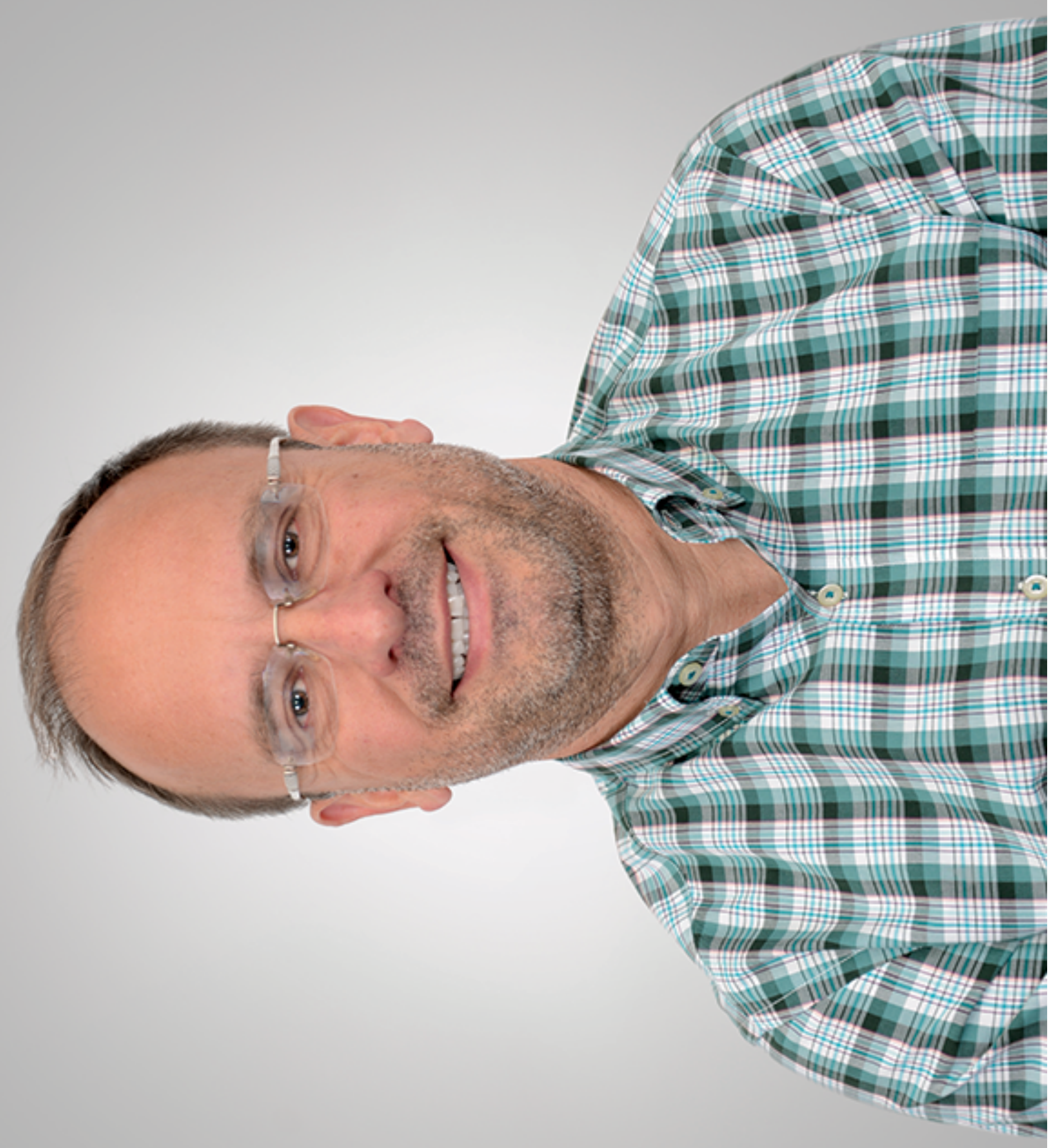




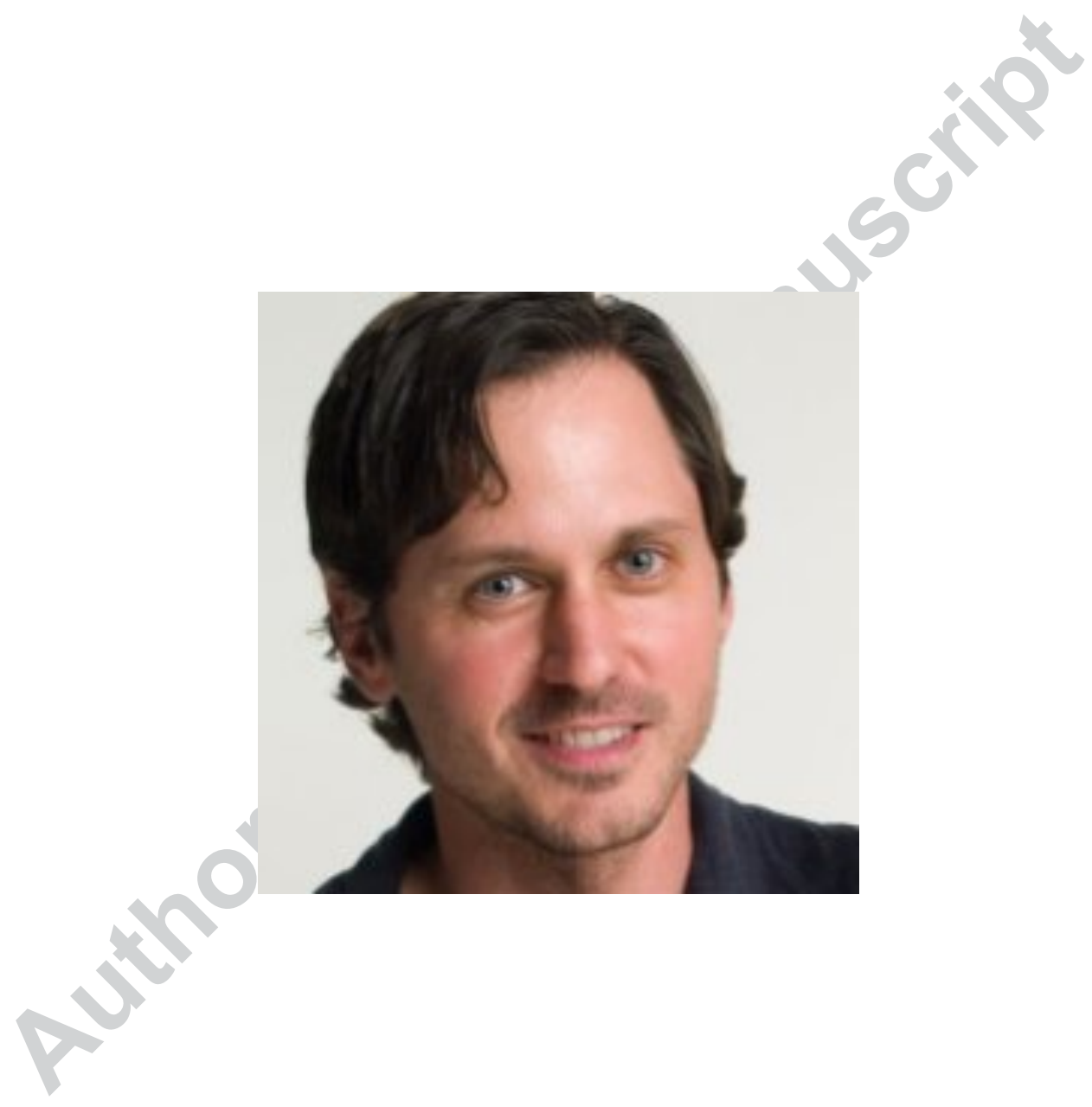

